\title{
Reliability and Validity of the Korean Version of Somatic Symptom Scale-8
}

\author{
Chan-Mo Yang ${ }^{1}$, Kyu-Sic Hwang ${ }^{1}$, Sang-Yeol Lee ${ }^{1}$, Jeong Seok Seo ${ }^{2}$, and Seung-Ho Jang ${ }^{1 凶}$ \\ ${ }^{1}$ Department of Psychiatry, School of Medicine, Wonkwang University, Iksan, Republic of Korea \\ ${ }^{2}$ Department of Psychiatry, School of Medicine, Konkuk University, Chungju, Republic of Korea
}

Objective Somatic symptoms in psychiatry include underlying depression, anxiety, or other psychiatric disorders. This study aimed to conduct a validation study of a Korean version of the Somatic Symptom Scale-8 (K-SSS-8), and to utilize the K-SSS- 8 effectively in clinical settings.

Methods For reliabilty, test-retest reliability and internal consistency were analyzed. For construct validity, exploratory factor analysis (EFA) and confirmatory factor analysis (CFA) were conducted. Known-group validity was verified, Jonckheere-Terpstra test (J-T statistic) were used.

Results Maternal Cronbach's alpha was 0.85 and $r$ value of test-retest reliability was 0.777 . In the EFA, 2-, 3- and 4-factor model showed cumulative percentile for variance of $60 \%$ or more. In the CFA, the 3-factor model was found to be the most appropriated and simplest $\left(\chi^{2}=10.992, \mathrm{df}=17, \mathrm{CFI}=1.000, \mathrm{TLI}=1.022, \mathrm{RMSEA}=0.000\right)$. The verifying the difference in $\mathrm{K}-\mathrm{SSS}-8$ also showed significant difference. ( $(\mathrm{J}-\mathrm{T}$ statistic $=-2.510, \mathrm{p}<0.05)$.

Conclusion K-SSS- 8 can be useful for exploring symptoms such as panic symptoms, physical pain, and physiological symptoms experienced by patients in a short time. In addition, the K-SSS-8 is expected to be very useful for determining the current severity by using the severity categories and for establish additionally required assessment plans for depression and anxiety symptoms.

Psychiatry Investig 2020;17(8):814-821

Key Words Somatic symptom, Patient Healh Questionnaire-15, Somatic Symptom Scale-8, Health anxiety.

\section{INTRODUCTION}

Somatic symptoms commonly include cardiopulmonary, gastrointestinal, pain, and general symptoms. ${ }^{1-3}$ Somatic symptoms in psychiatry include underlying depression, anxiety, or other psychiatric disorders. ${ }^{4-6}$ People with such somatic symptoms often visit the department of internal medicine rather than visiting the department of psychiatry first. Specifically, after patients visit the department of internal medicine

Received: March 24, 2020 Revised: May 28, 2020

Accepted: June 5, 2020

$\triangle$ Correspondence: Seung-Ho Jang, MD

Department of Psychiatry, School of Medicine, Wonkwang University, 895 Muwang-ro, Iksan 54538, Republic of Korea

Tel: +82-63-859-1044, Fax: +82-63-857-1043, E-mail: sh-jang82@hanmail.net

$\triangle$ Correspondence: Jeong Seok Seo, MD, PhD

Department of Psychiatry, School of Medicine, Konkuk University, 82 Gugwon-daero, Chungju 27376, Republic of Korea

Tel: +82-43-840-8470, Fax: +82-43-840-8982, E-mail: sjs52632@hanmail.net

(c) This is an Open Access article distributed under the terms of the Creative Commons Attribution Non-Commercial License (https://creativecommons.org/licenses/bync/4.0) which permits unrestricted non-commercial use, distribution, and reproduction in any medium, provided the original work is properly cited. and are told that they have no specific abnormal finding, they finally visit the department of psychiatry. When examining such patients, psychiatrists may consider the section on "Somatic Symptom and Related Disorders" in the Diagnostic and Statistical Manual of Mental Disorders, Fifth Edition (DSM-5) from a diagnostic perspective. ${ }^{7}$ In particular, doctors in charge needs to find out what somatic symptoms their patients have. In this case, the Patient Health Questionnaire-15 (PHQ-15) can be very useful. ${ }^{8}$

The PHQ is a tool that can easily screen depression, anxiety, alcohol, eating, and somatic symptom-related mental disorders when diagnosing patients. The PHQ has been translated into over 20 languages, and is widely used worldwide., ${ }^{9,10}$ In particular, the PHQ-15 is a PHQ version that can detect somatic symptoms, and is a tool for measuring the type and severity of somatic symptoms that patients are currently complaining of. In South Korea, the PHQ-15 was assessed in a validation study by Han and his colleagues, and has being utilized usefully so far. ${ }^{11}$ The Somatic Symptoms Scale-8 (SSS-8), which is intended to be verified in this study is a short form of the PHQ-15. 
The SSS- 8 is composed of a total of 8 items excluding items regarding menstrual problems, sexual problems, and fainting contained in the PHQ-15. It was originally developed by Kroenke et al., ${ }^{8}$ and known as the PHQ-Somatic Symptom Short-Form. ${ }^{12}$ However, the validation study for this tool was conducted by Gierk et al. ${ }^{13}$ in Germany, and its name was changed to SSS-8, accordingly. That study proved that the internal consistency of SSS- 8 was suitable, and revealed that it consisted of a 4-factor structure (gastrointestinal, pain, fatigue, and cardiopulmonary). In addition, that study classified severity into 5 categories, and proposed severity categories to easily check the severity of symptoms. Following this, a validation study for a Japanese version of the SSS-8 was conducted, and also verified its reliability and validity. ${ }^{14}$ Furthermore, that Japanese study verified the known-group validity, and explained somatic symptoms according to group. As found in the aforementioned previous studies, the SSS- 8 is a useful tool to assess somatic symptoms and severity of patients' complaints in a short time in the clinical settings. Despite the clinical usefulness of the SSS-8, the translation and validation of the SSS-8 has not been conducted in South Korea.

This study aimed to conduct a validation study of a Korean version of the SSS-8 (K-SSS-8), and to utilize the K-SSS-8 effectively in clinical settings.

\section{METHODS}

\section{Development of the Korean version of the Somatic Symptom Scale}

Before developing the K-SSS-8, we first obtained permission from the original authors of the SSS- 8 for the validation study of the Korean version. Subsequently, 3 psychiatrists and 1 clinical psychologist translated the SSS-8 into Korean, and an individual with a doctorate degree in the US, who are fluent in both English and Korean, back-translated the Korean version. After 2 psychiatrists and a psychologist with a $\mathrm{PhD}$ in psychology conferred, the K-SSS- 8 was finalized (Supplementary Materials in the online-only Data Supplement).

\section{Participants}

The study period was from March 2017 to March 2019. The participants consisted of healthy controls and patients. The healthy controls included 188 public officers working in smalland medium-sized cities in Jeollabuk-do, South Korea. Data from a total of 167 public officers, except for 11 with insufficient responses and 10 with serious medical conditions, were used for analysis. Among them, a total of 31 were retested for test-retest reliability testing after 3-4 weeks. In the case of the patient group, the participants were 32 patients who visited the Department of Psychiatry at our hospital and complained of somatic symptoms. Among them, data from a total of $23 \mathrm{pa}-$ tients, except for those with a high degree of psychosis and underlying serious and obvious medical conditions, were used for analysis. This study was approved by the Institutional Review Board of Wonkwang University Hospital (No. WKUH 2017-04-007-001), and all the participants provided written informed consent before starting the study.

\section{Measures}

The EuroQol 5 Dimension (EQ-5D), the PHQ-15, and the PHQ-2 scales were used to test the reliability and validity of the K-SSS-8.

\section{EuroQol 5 Dimension}

The EQ-5D was developed to measure general health status. ${ }^{15}$ The EQ-5D consists of a total of 5 items regarding mobility, self-care, usual activities, pain/discomfort, and anxiety/ depression, with responses measured using a 1-3 point-Likert scale. A higher score indicates poorer health status. A validation study of the Japanese version of SSS- 8 found that there was positively significant correlation between the EQ$5 \mathrm{D}$ and the Japanese version of the SSS- $8 .{ }^{14}$

\section{Patient Health Questionnaire-15}

The PHQ- 15 is a scale that consists of 15 items only related to somatic symptoms among all the items contained in the PHQ. Each item is rated on a 0-2-point scale, and the total score ranges from 0 to 30 points. In this study, the Korean version of the PHO-15 translated by Han et al., ${ }^{11}$ was used. The internal consistency of PHQ-15 was 0.834 in this study, showing a "good" internal consistency.

\section{Patient Health Questionnaire-2}

The PHQ-2 consists of two items regarding depression among the items contained in the PHQ-9. The two items ask about depression and anhedonia respectively. As in a study regarding the Japanese version of the SSS-8, a binary response consisting of "yes" or "no" was used in this study. If anyone responds with "yes" to at least one item, he or she is considered to have depression. Using this, the participants were divided into three groups according to the degree of depression (group 1: depression-positive for two items; group 2: depression-positive for one item; and group 3: depression-negative for two items), and the degree of somatic symptoms according to the group was analyzed.

\section{Statistical analysis}

First, frequency analysis was performed for descriptive statistics of the participants. Internal consistency and test-retest were used to validate reliability. Internal consistency was test- 
ed using Cronbach's alpha. Pearson correlation analysis was performed to verify test-retest. Construct validity and concurrent validity verification were performed to test validity. Exploratory factor analysis (EFA) and confirmatory factor analysis (CFA) were performed to verify construct validity, and correlation analysis was performed to verify concurrent validity. The Maximum Likelihood (ML) and Oblique rotation (Direct Oblimin) methods were used for the EFA, while Tucker-Lewis Index (TLI), Comparative Fit Index (CFI), and Root Mean Square Error of Approximation (RMSEA) among goodness-of-fit indices were used for the CFA. When TLI and CFI are 0.90 or higher, it is "acceptable, ${ }^{\text {"16,17 }}$ and when RMSEA is 0.08 or lower, it can be deemed "acceptable."18

Known-group validity was verified, and the participants were classified into three groups according to the responses to the PHQ-2 items (group 1: those who responded with "yes" to both of the two items; group 2: those who responded with "no" to any of the two items; and group 3: those who responded with "no" to the two items). Jonckheere-Terpstra test, a nonparametric test, was performed to verify the mean difference between the groups. ${ }^{19}$ Finally, frequency analysis according to score range was performed to classify the severity of the K-SSS-8. All statistical analyses were conducted with SPSS and AMOS 18.0 for windows (SPSS Inc., Chicago, IL, USA). The set level of significance was $5 \%$.

\section{RESULTS}

\section{Descriptive statistics of the sample}

Table 1 shows the demographic characteristics and descriptive statistics of the participants. The mean age of the healthy control group was 41.67 years. The age ranges from 26 to 63 . Of the participants, $23.4 \%$ were male, and $76.6 \%$ were female. The mean duration of education was 15.83 years. In addition, $28.1 \%$ were unmarried, and $69.5 \%$ were married. In terms of income level, the proportion of those with income of between KRW 2 million and KRW 3 million were the highest with $36.5 \%$. The proportions of smokers and non-smokers are $90.4 \%$ and $9.6 \%$, respectively.

Meanwhile, the mean age of the patient group was 55.95 years old, and $39.1 \%$ were male and $60.9 \%$ were female. The age ranges from 21 to 73 . The mean duration of education was 11.39 years. Among them, $8.7 \%$ were unmarried, and $73.9 \%$ were married. In terms of income level, the proportion of those with income of between KRW 1 million and KRW 2 million was the highest with $26.1 \%$. The proportions of smokers and non-smokers were $52.2 \%$ and $47.8 \%$, respectively.

The variables of age, years of education, gender, marital status, income level, and smoking status showed significant differences between the two groups [ $\mathrm{t}(188)=6.55, \mathrm{p}<0.001$ for
Table 1. Participants' demographic and clinical characteristics

\begin{tabular}{|c|c|c|c|}
\hline \multirow[b]{2}{*}{ Variable } & \multicolumn{2}{|c|}{ Group } & \multirow{2}{*}{$\frac{\text { Statistic }}{\mathrm{t}, \chi^{2}}$} \\
\hline & $\begin{array}{l}\text { Normal adults } \\
\qquad(\mathrm{N}=167)\end{array}$ & $\begin{array}{l}\text { Patient } \\
(\mathrm{N}=23)\end{array}$ & \\
\hline Age, years: mean \pm SD & $41.67 \pm 9.22$ & $55.95 \pm 13.37$ & $6.55^{\ddagger}$ \\
\hline Gender, male: $\mathrm{N}(\%)$ & $39(23.4 \%)$ & $9(39.1 \%)$ & 2.67 \\
\hline $\begin{array}{l}\text { Education, years: } \\
\text { mean } \pm S D\end{array}$ & $15.83 \pm 0.80$ & $11.39(3.81)$ & $13.25^{\ddagger}$ \\
\hline Marital status, N (\%) & & & $15.39^{\dagger}$ \\
\hline Unmarried & $47(28.1)$ & $2(8.7)$ & \\
\hline Married & $116(69.5)$ & $17(73.9)$ & \\
\hline Separated & $1(0.6)$ & $1(4.3)$ & \\
\hline Divorced & $2(1.2)$ & $1(4.3)$ & \\
\hline Lost & $1(0.6)$ & $2(8.7)$ & \\
\hline Income level (W) & & & $43.14^{\ddagger}$ \\
\hline Less than 1.0 million & $1(0.6)$ & $6(26.1)$ & \\
\hline $1.0-2.0$ million & $56(33.5)$ & $6(26.1)$ & \\
\hline 2.0-3.0 million & $61(36.5)$ & $3(13.0)$ & \\
\hline 3.0-4.0 million & $23(13.8)$ & $5(21.7)$ & \\
\hline $4.0-5.0$ million & $21(12.6)$ & $1(4.3)$ & \\
\hline More than 5.0 million & $5(3.0)$ & $2(8.7)$ & \\
\hline $\begin{array}{l}\text { Smoking/ } \\
\text { non-smoking, N (\%) }\end{array}$ & $\begin{array}{l}151(90.4) / \\
16(9.6)\end{array}$ & $\begin{array}{l}12(52.2) / \\
11(47.8)\end{array}$ & $24.26^{\ddagger}$ \\
\hline
\end{tabular}

${ }^{\dagger} \mathrm{p}<0.01,{ }^{\ddagger} \mathrm{p}<0.001$. N: number, SD: standard deviation

age; $\mathrm{t}(188)=13.25, \mathrm{p}<0.001$ for years of education; $\chi^{2}=15.39$, $\mathrm{p}<0.001$ for marital status; $\chi^{2}=15.39, \mathrm{p}<0.001$ for income level; $\chi^{2}=15.39, \mathrm{p}<0.001$ for smoking]. Since the purpose of this study is not a study for verifying the mean difference between the two groups, it is considered to be a study on the reliability and validity of the scale, so it seems that the differences in the demographic data does not have a significant effect on the validation study for the scale.

\section{Internal consistency and test-retest reliability}

The results of investigating internal consistency among reliability showed that the Cronbach's alpha of the K-SSS-8 was 0.85 . The verification of test-retest reliability was also found to be suitable $(r=0.777, \mathrm{n}=31, \mathrm{p}<0.001)$.

\section{Concurrent validity}

Table 2 shows the results of the correlation analysis between K-SSS-8 and EQ-5D, PHQ-2, and PHQ-15 to verify the concurrent validity of K-SSS-8. The K-SSS-8 showed significant correlation with all the three scales (For EQ-5D, $\mathrm{r}=0.493, \mathrm{n}=167, \mathrm{p}<0.001$; for PHQ-2, $\mathrm{r}=-0.226, \mathrm{n}=167$, $\mathrm{p}<0.01$; for PHQ-15, $\mathrm{r}=0.857, \mathrm{n}=167, \mathrm{p}<0.001$ ). 


\section{Construct validity}

First, it was found that factor analysis can be performed through Kaiser-Meyer-Olkin (KMO) values and Bartlett's test of sphericity $\left[\mathrm{KMO}=0.863 ; \chi^{2}(\mathrm{df}=28, \mathrm{n}=167)=476.348\right.$, $\mathrm{p}<0.001] .{ }^{20}$ The result of exploratory factor analysis showed that the 3-factor structure was found to be the most valid. The explanatory power of the 3 -factor structure was $71.59 \%$, and the RMSEA value was found to be suitable with 0.00 . The results of performing CFA to verify the goodness-of-fit of the 3-factor structure showed that the 3-factor structure was found to be suitable with TLI of 1.022, CFI of 1.000, and

Table 2. Correlation analysis of K-SSS- 8 with other somatic symptom scales for the concurrent validity $(\mathrm{N}=167)$

\begin{tabular}{lllll}
\hline & K-SSS-8 & PHQ-15 & EQ-5D & PHQ-2 \\
\hline K-SSS-8 & 1.00 & & & \\
PHQ-15 & $0.857^{\ddagger}$ & 1.00 & & \\
EQ-5D & $0.493^{\ddagger}$ & $0.502^{\ddagger}$ & 1.00 & \\
PHQ-2 & $-0.226^{\dagger}$ & $-0.171^{*}$ & $-0.219^{\dagger}$ & 1.00 \\
\hline
\end{tabular}

${ }^{*} \mathrm{p}<0.05,{ }^{\dagger} \mathrm{p}<0.01,{ }^{\ddagger} \mathrm{p}<0.001$. K-SSS-8: Korean version of Somatic Symptom Scale-8, PHQ-15: Patient Health Questionnaire-15, EQ5D: EuroQol 5 Dimension, PHQ-2: Patient Health Questionnaire-2
RMSEA of 0.00 . Table 3 shows the results of verification according to the number of factors. The first extracted factors included item \#6 ("Dizziness"), item \#4 ("Headaches"), and item \#5 ("Chest pain or shortness of breath"). The authors of this study named the first factor as "Cardiopulmonary." The second extracted factor was item \#2 ("Back pain"), and item \#3 ("Pain in your arms, legs, or joints"), which were named as "Pain." Lastly, the third factor extracted included item \#1 ("Stomach or bowel problems"), item \#7 ("Feeling tired or having low energy"), and item \#8 ("Trouble sleeping"), which were named as "Gastrointestinal and Fatigue." Table 4 shows each item and factor loading value according to factors, and Figure 1 shows items according to each factor name.

\section{Known-group validity}

The number of those who responded with "yes" to both items of the two of the PHQ-2 was 87 , accounting for $52.10 \%$. The number of those who responded with "no" to any item of the two was 22 , accounting for $13.2 \%$. In addition, the number of those who responded with "no" to both items was 58 , accounting for $34.7 \%$. The results of verifying the difference in the total score of K-SSS-8 between the three groups also showed significant difference (for Jonckheere-Terpstra test,

Table 3. The goodness-of-fit for the 2-, 3-, and 4-factor model by factor analysis solutions

\begin{tabular}{cccccccc}
\hline \multirow{2}{*}{ FAS } & Model & $\begin{array}{c}\text { Cumulative } \% \text { of } \\
\text { variance explained }\end{array}$ & $\chi^{2}$ & df & TLI & CFI & RMSEA \\
\hline EFA & 2-factor & 61.67 & 30.348 & 13 & N/A & N/A & 0.0896 \\
& 3-factor & 71.59 & 4.863 & 7 & N/A & N/A & 0.0000 \\
& 4-factor & 79.52 & 2.317 & 2 & N/A & N/A & 0.0309 \\
CFA & 2-factor & N/A & 35.104 & 19 & 0.948 & 0.965 & 0.0710 \\
& 3-factor & N/A & 10.992 & 17 & 1.022 & 1.000 & 0.0000 \\
& 4-factor & N/A & N/A & N/A & N/A & N/A & N/A \\
\hline
\end{tabular}

FAS: factor analysis solutions, EFA: exploratory factor analysis, CFA: confirmatory factor analysis, TLI: Tucker-Lewis index, CFI: comparative fit index, RMSEA: root mean square error of approximation

Table 4. EFA pattern matrix of the K-SSS-8 items for the 3-factor model

\begin{tabular}{|c|c|c|c|}
\hline Item no. & $\begin{array}{c}\text { Factor 1: } \\
\text { cardiopulmonary }\end{array}$ & $\begin{array}{l}\text { Factor 2: } \\
\text { pain }\end{array}$ & $\begin{array}{c}\text { Factor 3: } \\
\text { gastrointestinal and fatigue }\end{array}$ \\
\hline 06. Dizziness & 0.879 & & \\
\hline 04. Headaches & 0.681 & & \\
\hline 05. Chest pain or shortness of breath & 0.514 & & \\
\hline 03. Pain in your arm, legs, or joints & & -0.814 & \\
\hline 02. Back pain & & -0.744 & \\
\hline 01. Stomach or bowel problems & & & 0.834 \\
\hline 07. Feeling tired or having low energy & & & 0.585 \\
\hline 08. Trouble sleeping & & & 0.464 \\
\hline
\end{tabular}

EFA: exploratory factor analysis, K-SSS-8: Korean version of Somatic Symptom Scale-8, Factor 1: "Cardiopulmonary," Factor 2: "Pain,” Factor 3: "Gastrointestinal and Fatigue" 


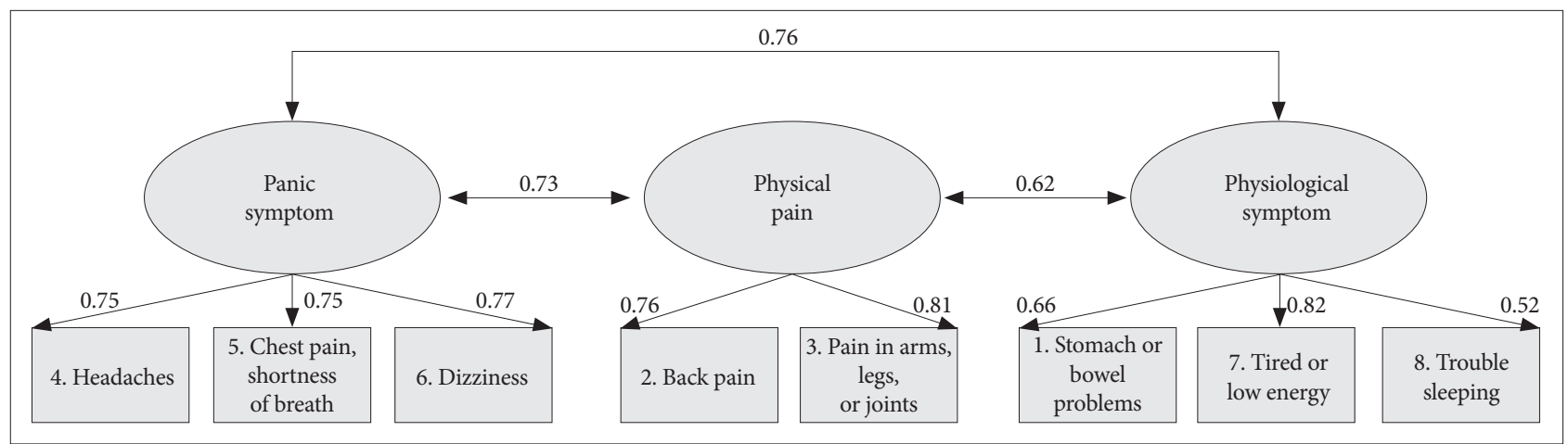

Figure 1. K-SSS-8 items by 3-factor model. K-SSS-8: Korean Version of Somatic Symptom Scale-8.

$\mathrm{J}-\mathrm{T}$ statistic $=-2.510, \mathrm{p}<0.05)$. Table 5 shows the results of verifying the mean difference in the total scores of the K-SSS-8, the PHQ-15, and the EQ-5D between the three groups.

\section{Severity categories}

As in previous studies, severity categories were set (a total of 5 categories), and the percentiles according to severity categories are shown in Table 5. A score of 0 to 3 points were classified as "no to minimal" severity, a score of 4 to 7 points as "low", a score of 8 to 11 points as "medium," a score of 12 to 15 points as "high," and a score of 16 points or higher as "very high." In the case of the healthy control group, more than half of the participants had "no to minimal" and "low" severity, and those with "high" and "very high" severity accounted for about $20 \%$. In the case of the patient group, the participants with "no to minimal" and "low" severity accounted for about 35\%, and those with "high" and "very high" severity accounted for about $43 \%$ (Table 6).

\section{DISCUSSION}

In this study, the SSS-8 was translated into Korean language for local adaption, the reliability and validity of its Korean version, the K-SSS- 8 was verified, and its clinical utility was investigated. The implications of the results are as follows.

First, the reliability analysis revealed that internal consistency and test-retest reliability were reliable. The internal consistency reliability of the K-SSS-8 was slightly better compared to previous studies (Cronbach's alpha $=0.81$ in a study by Gierk et al.). ${ }^{13}$ Test-retest reliability could not be compared because it had not been verified in previous studies. ${ }^{13,14}$ However, in this study, the test-retest reliability of the K-SSS- 8 was found to be a statistically reliable level. ${ }^{21}$ Taken together, the reliability of the K-SSS- 8 can be judged to be reasonably high.

Next, the results of verifying the goodness-of-fit of the number of factors in the validity analysis showed that the 3 -factor model was the most suitable. The explanatory vari-
Table 5. Testing result of known-group validity

\begin{tabular}{lccc}
\hline & Sum of K-SSS-8 & Sum of PHQ-15 & Sum of EQ-5D \\
\hline Group & Group 1, 2, 3 & Group 1, 2, 3 & Group 1, 2, 3 \\
J-T statistic & -2.510 & -1.670 & -2.729 \\
p & 0.012 & 0.095 & 0.006 \\
\hline
\end{tabular}

K-SSS-8: Korean version of Somatic Symptom Scale-8, PHQ-15: Patient Health Questionnaire-15, EQ-5D: EuroQol 5 Dimension, J-T: Jonckheere-Terpstra test

Table 6. The ratios according to K-SSS-8 severity in each group

\begin{tabular}{lcc}
\hline $\begin{array}{c}\text { K-SSS-8 severity } \\
\text { category (range) }\end{array}$ & $\begin{array}{c}\text { Normal adults } \\
(\mathrm{N}=167)\end{array}$ & $\begin{array}{c}\text { Patient } \\
(\mathrm{N}=23)\end{array}$ \\
\hline No to minimal $(0-3)$ & $48(28.7 \%)$ & $3(13.0 \%)$ \\
Low $(4-7)$ & $47(28.1 \%)$ & $5(21.7 \%)$ \\
Medium $(8-11)$ & $38(22.8 \%)$ & $5(21.7 \%)$ \\
High $(12-15)$ & $16(9.6 \%)$ & $3(13.0 \%)$ \\
Very high $(\geq 16)$ & $18(10.8 \%)$ & $7(30.4 \%)$ \\
\hline
\end{tabular}

K-SSS-8: Korean version of Somatic Symptom Scale-8

ance explained by the three factors in the 3-factor model was also more than $70 \%$, and the RMSEA value was less than 0.05 , indicating "excellent." ${ }^{18}$ In the EFA analysis, the 4-factor model showed an adequate goodness-of-fit, but in the CFA verifying the goodness-of-fit of the entire model, its goodness-of-fit was not satisfied. The reason is presumably thought to be due to the fact that when one factor was added from the 3-factor structure to the 4-factor structure, one item was assigned to the added factor. Because one factor can usually contain at least 2-3 items, ${ }^{5,22}$ the authors judged that it was not good in terms of economic feasibility and goodness-of-fit of the model when one item was generated as one factor. ${ }^{23}$ Meanwhile, this same problem occurred in the previous study by Gierk et al., ${ }^{13}$ but it seems that they selected a higher-order structure added with a general factor to solve with a 4-factor model. However, the authors of this study judged that the 3-factor model is adequate and concise for 8-item classification based on statistical theories. The implications of each factor in the 
3-factor model are as follows.

The first factor was named as "Cardiopulmonary," and included item \#6: “Dizziness," item \#4: "Headaches," and item \#5: "Chest pain or shortness of breath." In the study of Gierk et al., ${ }^{13}$ items \#5 and \#6 were grouped and expressed as cardiopulmonary symptoms. In this study, those 3 items were considered to be included in 13 types of Panic attack specifier in the DSM-5, and were thus named as "Cardiopulmonary." Recently, the number of people complaining of panic-like symptoms is increasing, and the number of patients with panic disorder in hospitals is also increasing. The first factor "Cardiopulmonary" is thought to be very useful in predicting people who are likely to develop panic disorder. So the first factor is likely that the mental health professionals will be able to identify symptoms easily, directly, and quickly. In particular, the items "Chest pain or shortness of breath" and "Dizziness" correspond to Panic disorder diagnosis criteria, so the factor can serve as evaluation items for diagnosis. On the other hand, the "Headaches" question is included, which is not consistent with the question of diagnose for the Panic disorder, but is a symptom that is often followed with Panic disorder. Unlike the findings of Gierk and his colleagues, the "Headaches" question was included in the "Cardiopulmonary" factor, perhaps due to differences in cultural background. When complaining of cardiopulmonary function problems, Koreans tend to complain of dizziness and headaches. It would be better to conduct a replication study on this part.

The second factor "Pain" included item \#3: "Pain in your arm, legs, or joints," and item \#2: "Back pain." The second factor literally means physical or body pain, and is thought to be common in patients with physical illness. In the study by Gierk et al., ${ }^{13}$ it was also named "Pain" factor. The second factor, "Pain" is thought to be very useful for detecting symptoms which appear on the surface of the body, such as the back and joints. The second factor includes pain in the back and joints, and when responding to questions related to "Pain," it seems that physical of surgical problems may be considered first. If the patient complains of symptoms related to the "Pain" factor even after excluding physical or surgical problems, the mental health professionals may consider "Somatic Symptoms and Related Disorders."

Finally, the third factor, "Gastrointestinal and Fatigue" included item \#1: "Stomach or bowel problems," item \#7: "Feeling tired or having low energy," and item \#8: "Trouble sleeping." The third factor is mainly related to physiological symptoms, which are often accompanied by complaints of somatic symptoms. This factor is thought to be closely related to digestive problems, sleep problems, and decreased vitality which are commonly observed in patients with depressive disorder, anxiety disorder, somatic symptoms, and related disor- ders. It is thought that the third factor enables us to quickly detect the presence or absence of physiological symptoms through the third factor. The third factor includes stomach and fatigue problems. From a psychiatric perspective, these problems are often accompanied by depression and anxiety. Therefore, there may be cognitive and emotional depression and anxiety at the basis of patients with gastrointestinal symptoms and fatigue, and in this case, it is recommended to conduct an additional scale related to depression and anxiety. Unlike Gierk et al. ${ }^{13}$ findings, the "stomach and bowel problems" item was included in the "fatigue" factor, which may be due to differences in cultural background. Koreans are known to be primarily accompanied by fatigue and gastrointestinal problems when stressed. Also, it would be better to have a replication study on this part.

In this study, Known-group validity was also verified using a Jonckheere-Terpstra test. According to the participants' responses (depression) to the PHQ-2, the participants were divided into three groups (group 1: depression-positive for both items; group 2: depression-positive for one item; and group 3: depression-negative for both items). The verification showed that there was a significant difference in the total KSSS- 8 score according to the degree of depression. Similarly, in terms of PHQ-15 and EQ-5D, there was a significance difference in the total scores between the groups. These results suggest that depression may be closely related to the complaint of somatic symptoms. ${ }^{6,24}$ It is known that $50-70 \%$ of patients with somatic symptoms and related disorders have a comorbid mental disorder, usually accompanied by depression and anxiety. ${ }^{7}$ Therefore, for patients complaining of somatic symptoms, it may be necessary to examine more thoroughly through interviews or measurements whether they have underlying depression or anxiety. ${ }^{25}$

In addition, frequency analysis according to the severity of the K-SSS-8 was performed for healthy control and patient groups in this study. In the case of the healthy control participants, the proportion of those who had higher than "medium" severity was $43.2 \%$, which is twice as high as in Japan (20.6\%). ${ }^{14}$ These results may be due to the fact that the participants were limited to public officers as the healthy control population, and it is thought that about half of these participants had at least 2-3 somatic symptoms.

In the case of the patient group, the proportion of those who had higher than "medium" severity was $65.1 \%$, unlike the control group, and about $2 / 3$ of the participants in the patient group complained of at least 2-3 somatic symptoms. Diagnostically, more than $90 \%$ of them had been diagnosed with depression or anxiety-related disorders, and had no medical abnormalities. It is known that a significant number of patients who complained of somatic symptoms without a 
medical condition initially visited the internal medicine departments. ${ }^{26}$ This suggests that consultation between internal medicine and psychiatry departments is required, and the importance of consultation. ${ }^{27}$

Taken together, in terms of clinical utility, the K-SSS- 8 can be useful for exploring symptoms such as panic symptoms, physical pain, and physiological symptoms experienced by patients in a short time. In addition, the K-SSS- 8 is expected to be very useful for determining the current severity by using the severity categories and for establish additionally required assessment plans for depression and anxiety symptoms. In particular, a K-SSS-8 score of 12 or higher is common in the patient group, but not common in the healthy control group. Therefore, "severe complaints of somatic symptoms" should be considered when establishing treatment plans.

The K-SSS- 8 is also thought to be useful in therapeutic aspects. Since the K-SSS-9 was divided into three factors (Cardiopulmonary, Pain, Gastrointestinal and Fatigue), it is thought that it will help to establish a pharmacotherapy or psychotherapy plan based on main symptoms. For example, it is known that the effect size of combined therapy (pharmacotherapy plus cognitive behavior therapy) is high for panic symptoms, and that when physiological symptoms are dominant, it is appropriate to consider pharmacotherapy first. ${ }^{28-30}$

Lastly, the limitations and future research directions of this study are as follows: First, the factor analysis revealed that the number ( $n=167)$ of the participant of this study was within an appropriate range, but it is recommended that the number (n) of participants is more than 200 participants to improve the power of a test. ${ }^{31}$ In addition, frequency analysis was performed with the data from the patient group, but it seems that it is desirable for the numbers of participants to be more than 50 so as to increase the power of a test. Second, severity levels could be identified using severity categories. However, cut-off scores are always used valuably in clinical settings. Therefore, it is considered that future studies are needed to investigate the total K-SSS-8 scores for patients with somatic symptoms and related disorders and to present cut-off scores through the receiver operating characteristic (ROC) analysis. Third, if complaints about somatic symptoms are common in patients with depression and anxiety disorders, it is considered necessary to perform K-SSS- 8 analysis according to such disorders. With regard to depression and anxiety disorders, related study findings such as the distribution of total scores, distribution of scores for each factor, and cut-off score estimation for each disorder through ROC analysis are thought to be very useful in actual clinical settings.

\section{Supplementary Materials}

The online-only Data Supplement is available with this ar- ticle at https://doi.org/10.30773/pi.2020.0112.

\section{Acknowledgments}

This study was supported by Wonkwang University in 2020.

\section{Conflicts of Interest}

The authors have no potential conflicts of interest to disclose.

\section{Author Contributions}

Conceptualization: Sang-Yeol Lee, Jeong Seok Seo, Seung-Ho Jang. Data curation: Sang-Yeol Lee, Kyu-Sic Hwang, Seung-Ho Jang. Formal analysis: Kyu-Sic Hwang, Seung-Ho Jang. Investigation: Chan-Mo Yang, Kyu-Sic Hwang, Seung-Ho Jang. Methodology: Jeong Seok Seo, Seung-Ho Jang. Project administration: Sang-Yeol Lee, Jeong Seok Seo, Seung-Ho Jang. Resources: Jeong Seok Seo, Seung-Ho Jang. Software: Kyu-Sic Hwang, Seung-Ho Jang. Supervision: Jeong Seok Seo, Seung-Ho Jang. Validation: Chan-Mo Yang, Sang-Yeol Lee, Seung-Ho Jang. Visualization: Kyu-Sic Hwang. Writing_original draft: Sang-Yeol Lee, Jeong Seok Seo, Seung-Ho Jang. Writing_review \& editing: Sang-Yeol Lee, Jeong Seok Seo, SeungHo Jang.

\section{ORCID iDs}

Chan-Mo Yang

Kyu-Sic Hwang

Sang-Yeol Lee

Jeong Seok Seo

Seung-Ho Jang https://orcid.org/0000-0002-4959-7595 https://orcid.org/0000-0002-8997-8720 https://orcid.org/0000-0003-1828-9992 https://orcid.org/0000-0001-9836-8425 https://orcid.org/0000-0002-3479-0552

\section{REFERENCES}

1. Fink P, Toft T, Hansen MS, Ørnbøl E, Olesen F. Symptoms and syndromes of bodily distress: an exploratory study of 978 internal medical, neurological, and primary care patients. Psychosom Med 2007;69: 30-39.

2. Witthöft M, Hiller W, Loch N, Jasper F. The latent structure of medically unexplained symptoms and its relation to functional somatic syndromes. Int J Behav Med 2013;20:172-183.

3. Zijlema WL, Stolk RP, Löwe B, Rief W, White PD, Rosmalen JG. How to assess common somatic symptoms in large-scale studies: a systematic review of questionnaires. J Psychosom Res 2013;74:459-468.

4. Kroenke K, Jackson JL, Judith Chamberlin PA M. Depressive and anxiety disorders in patients presenting with physical complaints: clinical predictors and outcome. Am J Med 1997;103:339-347.

5. Rief W, Martin A, Klaiberg A, Brähler E. Specific effects of depression, panic, and somatic symptoms on illness behavior. Psychosom Med 2005;67:596-601.

6. Simon GE, VonKorff M, Piccinelli M, Fullerton C, Ormel J. An international study of the relation between somatic symptoms and depression. N Engl J Med 1999;341:1329-1335.

7. American Psychiatric Association. Diganostic and Statistical Manual of Mental Disorders Fifth Edition. Washington D. C.: American Psychiatric Association; 2013.

8. Kroenke K, Spitzer RL, Williams JB. The PHQ-15: validity of a new measure for evaluating the severity of somatic symptoms. Psychosom Med 2002;64:258-266.

9. Spitzer RL, Kroenke K, Williams JB. Validation and utility of a self-report version of PRIME-MD: the PHQ primary care study. JAMA 1999;282:1737-1744.

10. Shin C, Lee SH, Han KM, Yoon HK, Han C. Comparison of the Usefulness of the PHQ-8 and PHQ-9 for Screening for Major Depressive Disorder: Analysis of Psychiatric Outpatient Data. Psychiatry Investig 2019;16:300-305.

11. Han C, Pae CU, Patkar AA, Masand PS, Kim KW, Joe SH, et al. Psychometric properties of the Patient Health Questionnaire-15 (PHQ- 
15) for measuring the somatic symptoms of psychiatric outpatients. Psychosomatics 2009;50:580-585.

12. Narrow WE, Clarke DE, Kuramoto SJ, Kraemer HC, Kupfer DJ, Greiner L, et al. DSM-5 field trials in the United States and Canada, Part III: development and reliability testing of a cross-cutting symptom assessment for DSM-5. Am J Psychiatry 2013;170:71-82.

13. Gierk B, Kohlmann S, Kroenke K, Spangenberg L, Zenger M, Brahler E, et al. The somatic symptom scale-8 (SSS-8): a brief measure of somatic symptom burden. JAMA Intern Med 2014;174:399-407.

14. Matsudaira K, Oka H, Kawaguchi M, Murakami M, Fukudo S, Hashizume M, et al. Development of a Japanese version of the Somatic Symptom Scale-8: Psychometric validity and internal consistency. Gen Hosp Psychiatry 2017;45:7-11.

15. Group TE. EuroQol-a new facility for the measurement of health-related quality of life. Health Policy 1990;16:199-208.

16. Hu Lt, Bentler PM. Cutoff criteria for fit indexes in covariance structure analysis: conventional criteria versus new alternatives. Struct Equ Modeling 1999;6:1-55.

17. Mulaik SA, James LR, Van Alstine J, Bennett N, Lind S, Stilwell CD. Evaluation of goodness-of-fit indices for structural equation models. Psychol Bull 1989;105:430-445.

18. Browne MW, Cudeck R. Alternative ways of assessing model fit. Sociol Methods Res 1992;21:230-258.

19. Terpstra TJ. The asymptotic normality and consistency of Kendall's test against trend, when ties are present in one ranking. Indagations Math 1952;14:327-333.

20. Kaiser HF. An index of factorial simplicity. Psychometrika 1974;39:31-36.
21. Bland JM, Altman D. Statistical methods for assessing agreement between two methods of clinical measurement. Lancet 1986;327:307-310.

22. Thompson B. Exploratory and Confirmatory Factor Analysis: Understanding Concepts and Applications. Washington, DC: American Psychological Association; 2004.

23. Mulaik SA. Parsimony and model evaluation. J Exp Educ 1998;66:266273.

24. Kapfhammer HP. Somatic symptoms in depression. Dialogues Clin Neurosci 2006;8:227-239.

25. Tylee A, Gandhi P. The importance of somatic symptoms in depression in primary care. Prim Care Companion J Clin Psychiatry 2005;7: 167-176.

26. Proust M. Overview: hypochondriasis, bodily complaints, and so-matic styles. Am J Psychiatry 1983;140:273-283.

27. Hwang KS, Jang SH, Lee HJ, Lee SY. Reliability and validity of the Korean version of health anxiety questionnaire. Psychiatry Investig 2018; 15:976-983

28. Gould RA, Ott MW, Pollack MH. A meta-analysis of treatment outcome for panic disorder. Clin Psychol Rev 1995;15:819-844.

29. Roy-Byrne P, Stein M, Bystrisky A, Katon W. Pharmacotherapy of panic disorder: proposed guidelines for the family physician. J Am Board Fam Pract 1998;11:282-290.

30. Zugliani MM, Cabo MC, Nardi AE, Perna G, Freire RC. Pharmacological and neuromodulatory treatments for panic disorder: clinical trials from 2010 to 2018. Psychiatry Investig 2019;16:50-58.

31. Comrey AL, Lee HB. A First Course in Factor Analysis. East Sussex: Psychology Press; 2013. 


\section{SUPPLEMENTARY MATERIAL}

Korean version of Somatic Symptom Scale-8 (K-SSS-8)

한국판 신체증상척도-8

\begin{tabular}{|c|c|c|c|c|c|c|}
\hline \multicolumn{7}{|c|}{ 지난 일주일 동안에 아래의 증상들로 인해 얼마나 많이 힘들었습니까? } \\
\hline & 0 & 2 & & & & \\
\hline & 전혀 그렇지 않다 & 다소 그렇다 & & 렇다 & 매두 & 그렇다 \\
\hline \multicolumn{2}{|r|}{ 문 항 } & \multicolumn{5}{|c|}{ 응 답 } \\
\hline 1 & 위통과 배변 문제 & 0 & 1 & 2 & 3 & 4 \\
\hline 2 & 허리 통증 & 0 & 1 & 2 & 3 & 4 \\
\hline 3 & 팔, 다리, 또는 관절의 통증 & 0 & 1 & 2 & 3 & 4 \\
\hline 4 & 두통 & 0 & 1 & 2 & 3 & 4 \\
\hline 5 & 가슴 통증 또는 숨이 가쁨 & 0 & 1 & 2 & 3 & 4 \\
\hline 6 & 어지러움 & 0 & 1 & 2 & 3 & 4 \\
\hline 7 & 피로감 또는 기운이 없음 & 0 & 1 & 2 & 3 & 4 \\
\hline 8 & 잠드는데 어려움 & 0 & 1 & 2 & 3 & 4 \\
\hline
\end{tabular}

